\title{
COPROCESSED EXCIPIENTS OF CROSSLINKED AMYLOSE AND XANTHAN GUM FOR USE IN CONTROLLED RELEASE DOSAGE FORMS
}

\author{
SILVIA SURINI*, LUSIANA ARIANI, KURNIA SS PUTRI, HAYUN HAYUN, EFFIONORA ANWAR
}

Laboratory of Pharmaceutics and Pharmaceutical Technology Development, Faculty of Pharmacy, Universitas Indonesia, Depok, Indonesia. Email: silvia@farmasi.ui.ac.id

Received: 06 June 2018, Revised and Accepted: 08 October 2018

\section{ABSTRACT}

Objective: This study was aimed to obtain a new excipient that can be used as a polymer matrix for the formulation of controlled release dosage forms.

Methods: This study used coprocessing and crosslinking methods on amylose and xanthan gum (XG) to obtain a new excipient that can be used for controlled release matrix of pharmaceutical dosage forms. The coprocessing step was conducted by drum drying, and the crosslinking step was prepared using 6 and 12\% sodium trimetaphosphate (STMP). The produced novel excipients were characterized in terms of infrared (IR) spectrum, substitution degree, moisture content, swelling index, and gel strength.

Results: Our results showed that amylose-XG excipients crosslinked using 6\% STMP have greater gel strength and better swelling indexes than excipients crosslinked using $12 \%$ STMP. All coprocessed excipients exhibited no differences in their IR spectra, whereas the crosslinked excipients did, indicating a structural change due to the addition of phosphate groups. Crosslinking amylose-xanthan-coprocessed excipients using 6\% STMP produced degrees of substitution (DSs) of 7-8 phosphates per 100 monomeric subunits. The excipients had a moisture content of $8.21-12.85 \%$, and the $\mathrm{pH}$ of a $1 \%$ solution of excipients was 6.21-6.43. In addition, the swelling index and gel strength of the excipient where both amylose and XG were crosslinked together Were more than 1 where only amylose was crosslinked.

Conclusion: The crosslinking amylose-xanthan-coprocessed excipient using 6\% STMP is more suitable for use in controlled release dosage forms, particularly when the polymer ratio is $1: 1$.

Keywords: Coprocessing, Crosslinking, High-amylose starch, Xanthan gum, Controlled release dosage forms.

(C) 2018 The Authors. Published by Innovare Academic Sciences Pvt Ltd. This is an open access article under the CC BY license (http://creativecommons. org/licenses/by/4. 0/) DOI: http://dx.doi.org/10.22159/ijap.2018.v10s1.13

\section{INTRODUCTION}

Polysaccharides such as pectin, chitosan, starch, guar gum, and xanthan are commonly used in controlled release dosage forms of various pharmaceuticals due to their low cost, biocompatibility, low toxicity, ease of chemical modification, and biodegradability [1-4]. Among the various types of commercially available starch, high-amylose starch has been demonstrated to possess superior properties with regard to drug delivery compared with native starch [5]. Xanthan gum (XG) has also been studied as an ingredient for producing a direct compression matrix with high swelling properties $[6,7]$.

Polymer modification can improve the physical and functional properties of each constituent polymer. Crosslinking has been proven to improve various characteristics of excipients such as swelling properties, solubility at various $\mathrm{pHs}$, gel strength, mucoadhesive properties, and resistance to enzymatic modification or degradation. Many studies have demonstrated the successful application of high-amylose starch crosslinked using different agents, such as epichlorohydrin and sodium trimetaphosphate (STMP), in the development of controlled drug delivery systems $[5,8,9]$. Lenaerts et al. have demonstrated that crosslinked amylose (CLA) can be used in controlled drug release systems over periods of 18-24 h [10]. CLA with degrees of substitution (DSs) of 6-8 exhibits a good release profile when used as matrix in controlled-release tablets (15-20 h); in contrast, high DS $(>10)$ results in a significant decrease in the drug release time profile $(1-3 \mathrm{~h})$, indicating that substitution can cause it to act as a disintegrants [11]. Meanwhile, according to Bejenariu et al., crosslinking using STMP increases the gel strength of $\mathrm{XG}$, which can then be used in controlled drug release systems [12].
Coprocessing is a new concept of manipulating the physical interaction of two or more excipients at the particulate level. The advantage of this method is that it can improve flow properties and compressibility and reduce weight variations by producing a single excipient with multiple functions. This can minimize the use of multiple excipients, produce excipients with improved functional properties with respect to viscosity or gel strength, and overcome the undesirable properties of the individual excipients to produce strong tablets with low compression pressure [13-15].

Based on these ideas, in this study, we modified high-amylose starch and XG using two methods. In the first, high-amylose starch was crosslinked using STMP and then coprocessed (Co) with XG. In the second, amylose and $X G$ were coprocessed and then crosslinked using STPM to produce Co CLA-XG.

Coprocessed excipients consisting of XG and CLA were used as in the preparation of a sustained release matrix. Sustained release dosage forms have the advantages of reducing the frequency of drug administration, increasing patient compliance, minimizing drug level fluctuations in plasma, producing constant pharmacological effects (especially for drugs with short biological half-lives), and reducing the drug's side effects.

Sodium diclofenac is a nonsteroidal anti-inflammatory drug that is often used to treat a variety of pain, such as that due to migraines and osteoarthritis. This drug has a short half-life of 1-2 $\mathrm{h}$ and oral bioavailability of approximately $50 \%$ due to the first-pass metabolism in the liver. Frequent dosing is used for long-term treatment for osteoarthritis. For these reasons, sodium diclofenac was chosen as a model drug in this study as a slow release of the drug would be expected 
to reduce administration frequency of the drug and provide constant pharmacological effects.

\section{MATERIALS AND METHODS}

\section{Materials}

Sodium diclofenac (Kimia Farma, Indonesia); high-amylose starch (Shangqiu Kangmedia Bio-Tech, China); XG (CV. Tristars Chemicals, Indonesia); STMP (Shangqiu Kangmedia Bio-Tech, China); sodium hydroxide (Merck, Jerman); chloride acid (Merck, Jerman); 96\% ethanol (Brataco, Indonesia); and distilled water (Brataco, Indonesia) were used.

\section{Methods}

\section{Synthesis of Co CLA-XG and CL Co-A-XG}

Two methods, which were modified from the method of Cury et al. [16], were used. In the first, amylose was first crosslinked and then coprocessed with XG to produce Co CLA-XG. Amylose was crosslinked using STMP after adjusting the $\mathrm{pH}$ to $11-12$ by adding $4 \% \mathrm{NaOH}$. STMP was used at concentrations of $6 \%$ or $12 \%$, and the reaction time was 1-4 h. Once the reaction was completed, the amylose suspension was neutralized using $\mathrm{HCl}$ to $\mathrm{pH} \pm 6$ and then washed with $96 \%$ ethanol. The CLA was then air-dried and sieved using a 35-mesh sieve. CLA and XG were then dispersed in distilled water, homogenized for $15 \mathrm{~min}$, and then dried in a drum drier at $80^{\circ} \mathrm{C} \pm 5^{\circ} \mathrm{C}$. The powder was then milled and sieved through a 35-mesh sieve. Three combinations of CLA and XG were produced at $1: 1,1: 2$, and $2: 1$.

In the second method, amylose and XG were first coprocessed and then crosslinked to produce CL Co-A-XG. A dispersion of amylose and XG in distilled water was homogenized for $15 \mathrm{~min}$ and then dried using drum drier at $80^{\circ} \mathrm{C} \pm 5^{\circ} \mathrm{C}$. The powder was then milled and sieved through a 35-mesh sieve. Three combinations of amylose and XG were produced at $1: 1,1: 2$, and $2: 1$. This material was suspended in distilled water, and the $\mathrm{pH}$ was adjusted to $11-12$ with $4 \% \mathrm{NaOH}$. STMP was added at a concentration of $6 \%$ or $12 \%$ and reacted for $1-4 \mathrm{~h}$. Once the reaction was completed, the suspension was neutralized using $\mathrm{HCl}$ to $\mathrm{pH} \pm 6$ and then washed with $96 \%$ ethanol. The final material was then air-dried and sieved using a 35-mesh sieve.

\section{Characterization of Co CLA-XG and CL Co-A-XG}

Physical characterization

Physical characterization of Co CLA-XG and CL Co-A-XG included its morphology, thermal properties, powder flowability, moisture content, hygroscopicity, and solubility. The shape and morphology of the powders were assessed using a scanning electron microscope. Thermal analysis was conducted using thermogravimetry-differential thermal analysis. Crystallinity was measured by X-ray diffraction. Moisture content was measured with a moisture balance using $1 \mathrm{~g}$ of sample powder, which was then heated to $105^{\circ} \mathrm{C}$. Hygroscopicity testing was conducted on a 1-g sample placed in a desiccator at room temperature and a humidity of $70 \%$ relative humidity. The sample was divided into four different containers and changes in weight before and after treatment were recorded.

Chemical characterization

Chemical characterization of Co CLA-XG and CL Co-A-XG was conducted with respect to $\mathrm{pH}$, number of functional groups, and degree of crosslinking. The excipient was dissolved in distilled water at a concentration of $10 \%$ and $\mathrm{pH}$ was measured using a $\mathrm{pH}$ meter. Substitution of phosphate groups in starch was ascertained using infrared (IR) spectrophotometer. IR spectra of Co CLA-XG and CL CoA-XG were compared with those of amylose and XG. Phosphate groups exhibited specific absorption by ester bonds at wave numbers 1298.14 and $2362.88 \mathrm{~cm}^{-1}$. The DS was measured using colorimetry.

\section{Functional characterization}

Functional characterization of Co CLA-XG and CL Co-A-XG powders was conducted on their flow properties, whereas viscosity and rheology, swelling index, and gel strength were assessed on their solutions/gels. The flow properties were measured with respect to flow rate, angle of repose, Hausner ratio, and compressibility index. Viscosity and rheology of Co CLA-XG and CL Co-A-XG were determined using their solutions at concentrations of $3 \%, 5 \%, 7 \%$, and $10 \% \mathrm{w} / \mathrm{v}$ using a Brookfield viscometer. Swelling index was determined by comparing the weight of a dry tablet of excipient and the weight after it had been placed in $10 \mathrm{~mL}$ of the medium of various $\mathrm{pHs}$. Gel strength was measured using a texture analyzer on a solid gel of 10\% Co CLA-XG or CL Co-A-XG that has been cooled to $\pm 4^{\circ} \mathrm{C}$ for $2 \mathrm{~h}$.

\section{RESULTS AND DISCUSSION}

Synthesis of Co CLA-XG and CL Co-A-XG

Crosslinking was achieved by reacting a $3 \%$ mucilago of each polymer in distilled water with $6 \%$ of STMP. The $\mathrm{pH}$ was adjusted to $11-12$ by continuous addition of $10 \mathrm{~N} \mathrm{NaOH}$ solution. The hydroxyl groups of amylose and XG were ionized under alkaline conditions and could react with the phosphate groups.

The reaction was conducted while stirring at $3000 \mathrm{rpm}$ in a homogenizer, and the crosslinking reaction is considered complete if there is no longer any change in the $\mathrm{pH}$ of the solution. The solution was then neutralized by adding $\mathrm{HCl}$ till a $\mathrm{pH}$ of $6.5-7$ is reached.

A $50 \%$ ethanol solution was added to the neutralized suspension to form a white precipitate. The precipitate was then washed with $50 \%$ ethanol until no phosphate was detected in the final rinse (identified by an absorption peak at $820 \mathrm{~nm}$ ). The precipitate was dried using a drum drier at a temperature of $80 \pm 5^{\circ} \mathrm{C}$, milled using a disc mill, and sieved through a 60-mesh sieve to obtain Co CLA-XG or CL Co-A-XG.

\section{Characterization of Co CLA-XG}

\section{Physical properties}

CLA consisted of fine, white granules, whereas Co CLA-XG consisted of non-porous yellowish white fine flakes/fibers. The flake/fiber morphology of Co CLA-XG was due to the drying process with a double drum drier that broke the starch granules and turned them into irregular thin flakes (Fig. 1)

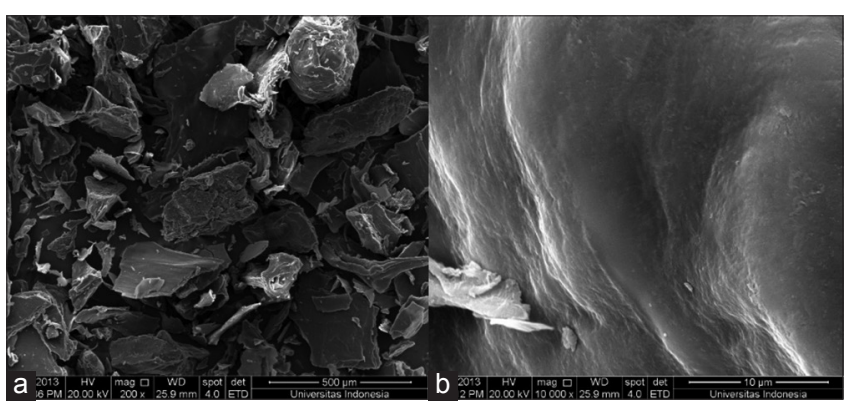

Fig. 1: Morphology of Co CLA-XG at $\times 200$ (a) and $\times 10,000$ magnifications (b)

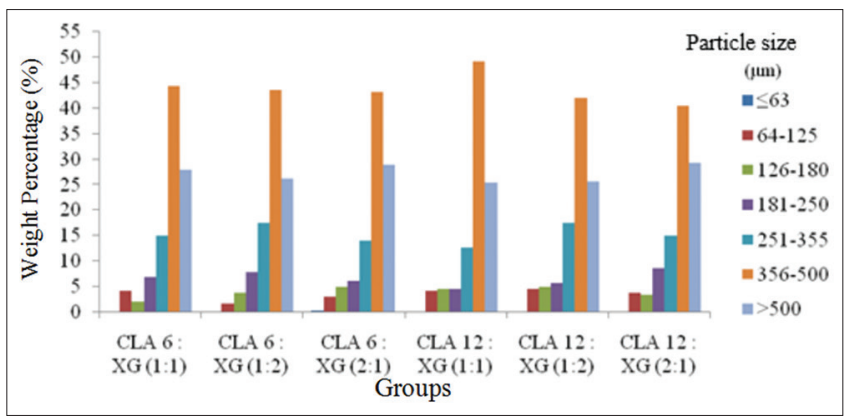

Fig. 2: Particle-size distribution of Co CLA-XG powders 
The most common particle size of the various excipient powders was 356-500 $\mu \mathrm{m}$, as shown in Fig. 2.

Table 1 shows that the moisture content of CLA was higher than that of Co CLA-XG. This was due to differences in drying methods; Co CLA$\mathrm{XG}$ was dried using a drum drier at a higher temperature than CLA, which was dried at room temperature. Nevertheless, all excipients contained $<15 \%$ water and flowed well (Table 2 ), so they could be used as excipients in pharmaceutical dosage forms.

Morphology, particle size, and moisture content contribute to the flow properties of powder excipients. Table 2 shows that all versions of Co CLA-XG performed well. High water levels can also cause poor flow rates due to adhesion between particles to form larger particles. However, while CLA6 had a higher water content, its spherical granules resulted in a good flow rate.

\section{Chemical properties}

Amylose was first crosslinked using STMPat two different concentrations (6\% and 12\%) before Co with XG. Crosslinking produced CLA with different DSs: 0.07 and 0.11 (Table 3). This means that there are approximately 7 or 11 phosphate groups for every 100 anhydroglucose units in amylose depending on the STMP concentration. Crosslinking is expected to occur at $\mathrm{C}$ positions 2, 3, and 6 of the starch, with a higher probability at the $\mathrm{C} 2$ position because it has the highest reactivity [17].

pH measurement on $1 \%$ solutions of the various Co CLA-XGs showed that they had a pH close to neutral (Table 4). This shows that there was no/very little residual phosphate in this crosslinked excipient and indicates that it is safe to use since its $\mathrm{pH}$ is close to physiological $\mathrm{pH}$.

Analysis of the functional groups of CL6 was performed using FTIR. This showed a specific absorption for P (0) 0 at $1298.14 \mathrm{~cm}^{-1}$, a peak for oxygen single bonds (-PO) of the phosphate group at $933.58 \mathrm{~cm}^{-1}$, and an oxygen double bond $(-\mathrm{P}=0)$ at $1062.81 \mathrm{~cm}^{-1}$. Amylose and CLA6 produced different spectra between 4000 and $2000 \mathrm{~cm}^{-1}$. The additional peak at $2362.88 \mathrm{~cm}^{-1}$ in CLA6 that was due to the phosphate groups from STMP has reacted with amylose, while the loss of the peak at $2945.40 \mathrm{~cm}^{-1}$ in CLA6 was due to the partial loss of hydroxyl groups through reaction with STMP.

Analysis of the functional groups of the Co-CLA6-XG was performed to identify the physical interactions between XG and CLA6. The absence of a new group that emerged proving that there were no chemical interactions.

\section{Functional properties}

Co CLA6-XG exhibited greater gel strength than Co CLA12-XG, suggesting that Co-CLA6 PVA might be more suitable as a controlled release matrix (Fig. 3)

Table 1: Moisture content of Co CLA-XG

\begin{tabular}{ll}
\hline Excipient & Water content (\%) \\
\hline CLA6 & $12.58 \pm 0.62$ \\
CLA6:XG $(2: 1)$ & $8.21 \pm 1.11$ \\
CLA6:XG $(1: 1)$ & $8.22 \pm 1.08$ \\
CLA6:XG $(1: 2)$ & $8.41 \pm 0.94$ \\
\hline
\end{tabular}

Swelling index measurements were performed in distilled water and phosphate buffer at $\mathrm{pH}$ 7.4. Co CLA-XG exhibited greater swelling in water than in buffer. All Co CLA-XG variants swelled to 2 times their original volume in the first $90 \mathrm{~min}$, suggesting that the Co excipients could potentially be used as slow-release dosage forms. Co CLA6-XG $1: 1$ was the most promising excipient for use as a matrix with the ability to better retain and then release drugs. Results can be seen in Fig. 4.

Low viscosity is an indicator of a low gel strength, which can influence drug release. Co CLA-XG dispersed in distilled water exhibited pseudoplastic thixotropic flow properties, meaning that the viscosity of the solution decreased as the shear rate increased; however, when share rate is low, the viscosity of the solution does not immediately return to normal (Fig. 5).

\section{Characterization of CL Co- $A-X G$}

Physical properties

All Co-A-XG formulations were yellowish white flakes/fibers caused using a drum drier. In contrast, Co CLA-XG was non-porous, yellowish white, and granule-shaped. Differences in the shape of these modified excipients could impact their functional properties, such as flow rate and density. Morphology of CL Co-A-XG can be seen in Fig. 6.

Most of the powder particles were in the size range of $356-500 \mu \mathrm{m}$, as shown in Fig. 7.

Moisture content of Co A-XG was higher than that of CL Co-A-XG due to the different drying processes (Table 5). Co-A-XG was dried using the drum drying method; thus, its water content was lower than that of CL Co-A-XG, which was dried at room temperature.

Shape, size, and water content of the excipients impact the flow rate of the powder. Powder flow properties of all powders were in the fine category and their flow rates were quite good (Table 6).

\section{Chemical properties}

Crosslinking of Co-A-XG using STMP at concentrations of $6 \%$ and $12 \%$ resulted DSs listed in Table 7. Crosslinking of Co-A-XG with 6\% STMP produced CL Co-A-XG with a DS of 0.08 while crosslinking using $12 \%$ STMP produced a DS of 0.11-0.12.

All the CL Co A-XG excipients at 1\% concentration exhibited pHs close to neutrality (Table 8); however, all CL Co A-XG excipients had slightly

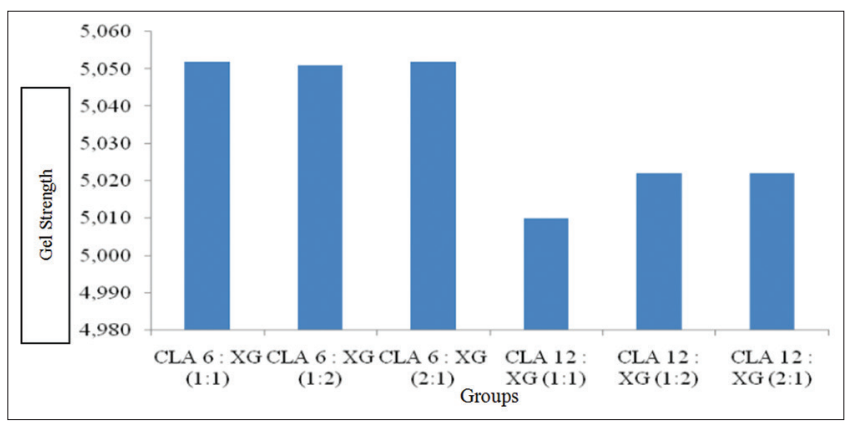

Fig. 3: Gel strength of Co CLA-XG

Table 2: Flow properties of Co CLA-XG

\begin{tabular}{llll}
\hline Excipient & Flow rate (g/det) & Tapped density (g/ml) & Compressibility index (\%) \\
\hline CLA6:XG (1:1) & 4.08 & 0.89 & 25.00 \\
CLA6:XG (1:2) & 4.05 & 0.87 & 24.59 \\
CLA6:XG (2:1) & 3.97 & 0.87 & 23.33 \\
CLA12:XG (1:1) & 4.17 & 0.89 & 25.00 \\
CLA12:XG (1:2) & 4.00 & 0.89 & 25.00 \\
CLA12:XG (2:1) & 4.14 & 0.89 & 25.00 \\
\hline
\end{tabular}




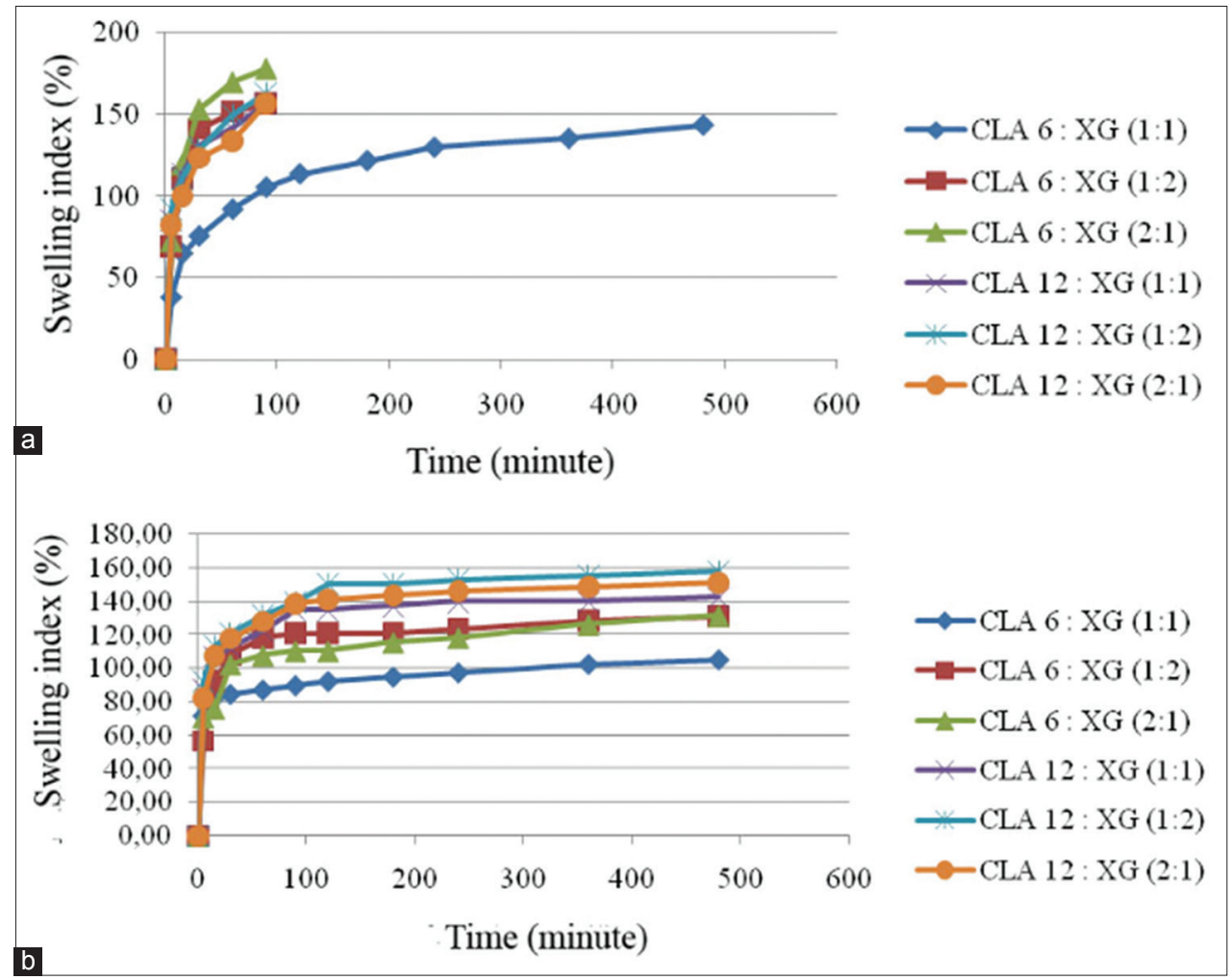

Fig. 4: Swelling indices of Co CLA-XG in (a) distilled water and (b) phosphate buffer at pH 7.4

Table 3: Degree of substitution of crosslinked amylose

\begin{tabular}{lll}
\hline Excipient & \% p & DS \\
\hline CLA6 & $1.43 \pm 0.175$ & $0.07 \pm 0.010$ \\
CLA12 & $2.12 \pm 0.418$ & $0.11 \pm 0.025$ \\
\hline
\end{tabular}

Table 4: pH of Co CLA-XG

\begin{tabular}{ll}
\hline Sample (1\%) & pH \\
\hline CLA6:XG (1:1) & $6.44 \pm 0.03$ \\
CLA6:XG (1:2) & $6.42 \pm 0.04$ \\
CLA6:XG (2:1) & $6.44 \pm 0.02$ \\
CLA12:XG (1:1) & $6.37 \pm 0.03$ \\
CLA12:XG (1:2) & $6.39 \pm 0.05$ \\
CLA12:XG (2:1) & $6.32 \pm 0.06$ \\
\hline
\end{tabular}

Table 5: Moisture content of excipient

\begin{tabular}{ll}
\hline Excipient & Moisture content (\%) \\
\hline Co-A-XG (1:1) & $9.31 \pm 1.02$ \\
Co-A-XG (1:2) & $9.58 \pm 1.00$ \\
Co-A-XG (2:1) & $9.12 \pm 0.75$ \\
Co CLA-XG (1:1) & $12.65 \pm 0.41$ \\
Co CLA-XG (1:2) & $12.85 \pm 0.46$ \\
Co CLA-XG (2:1) & $12.83 \pm 0.69$ \\
\hline
\end{tabular}

higher pHs than their non-crosslinked counterparts. Nevertheless, all the excipients produced were considered to be compatible for use in formulations because they were close to the physiological $\mathrm{pH}$ of the body.

There was a difference in the IR spectra of Co A-XG and CL Co A-XG, indicating that crosslinking had altered the chemical structure of the excipient.

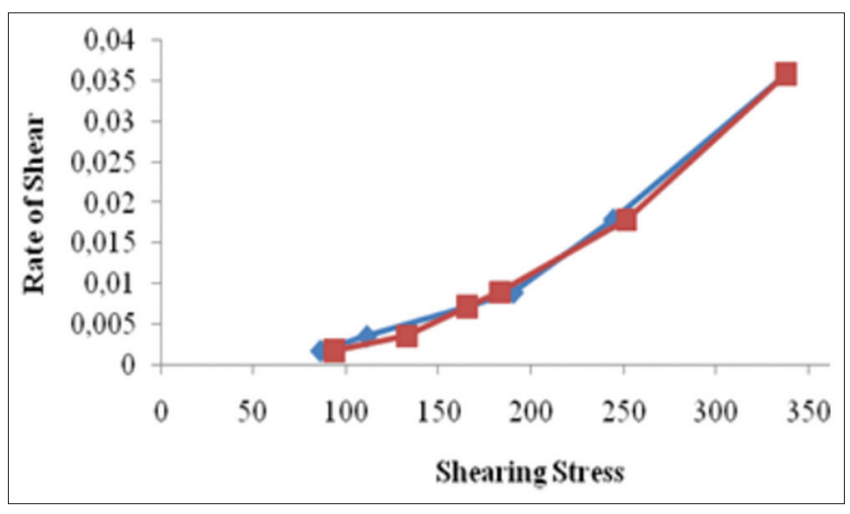

Fig. 5: Viscosity of Co CLA-XG

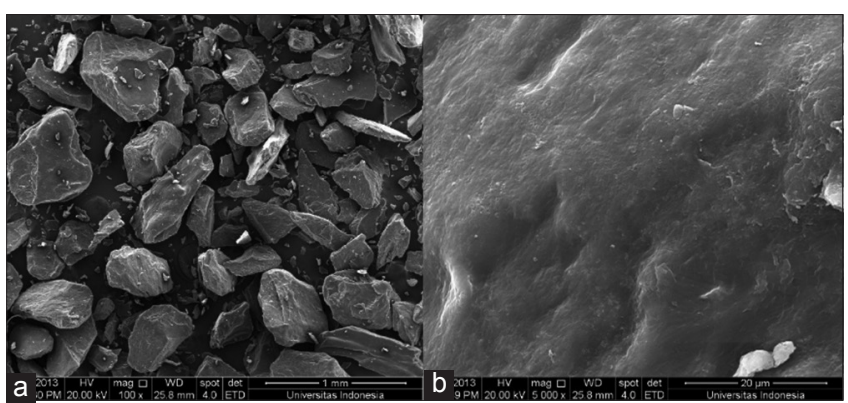

Fig. 6: Morphology of CL Co-A-XG at 100× (a) and 5000x magnifications (b)

Chemical properties

CL Co A-XG showed more swelling in the buffer than in distilled water (Fig. 8) and expanded up to $800 \%$ in the buffer (10 times higher than that in distilled water). 


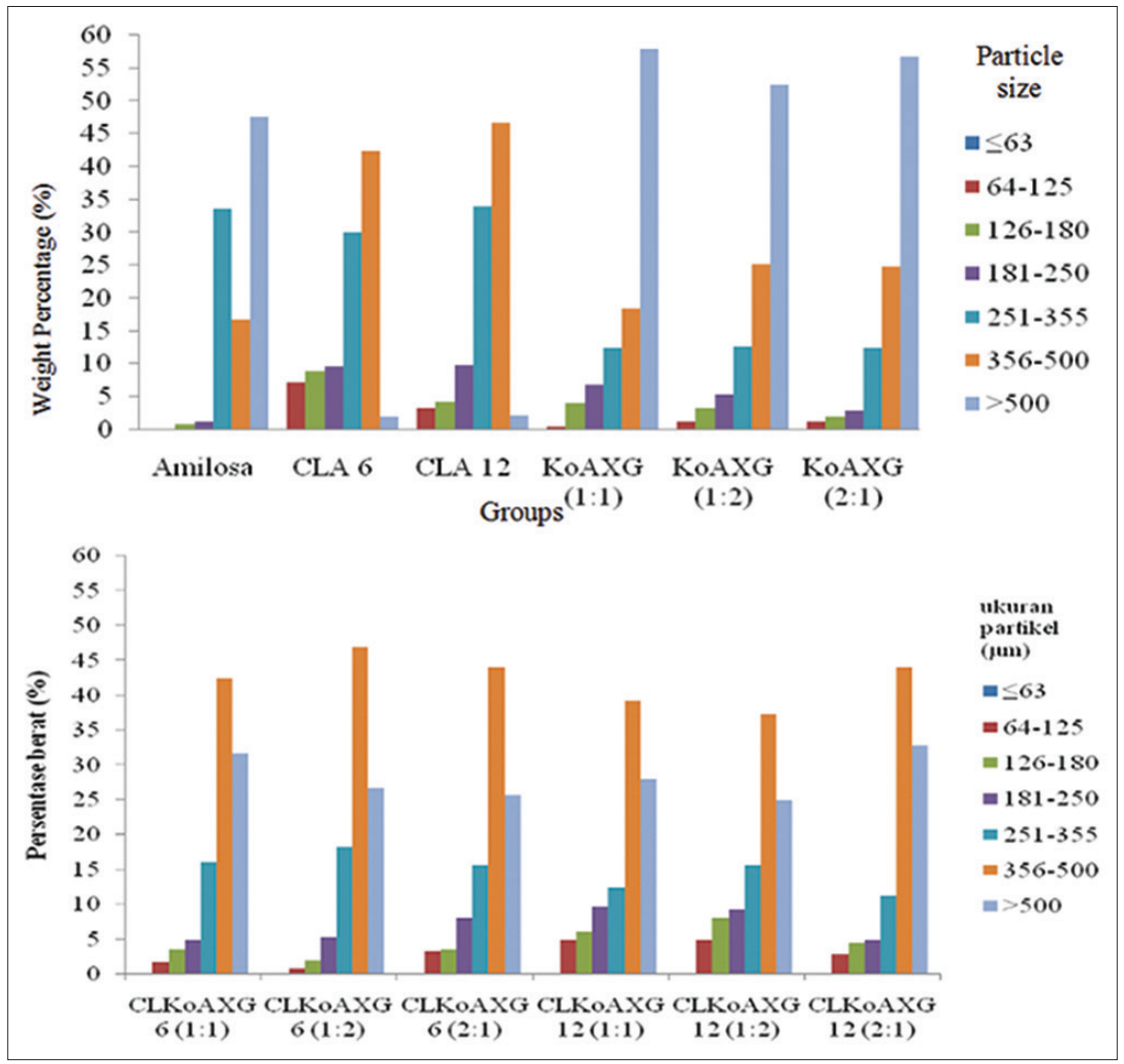

Fig. 7: Particle size distribution of coprocessed amylose-xanthan gum (Co-A-XG) (above) and CL Co-A-XG (below)

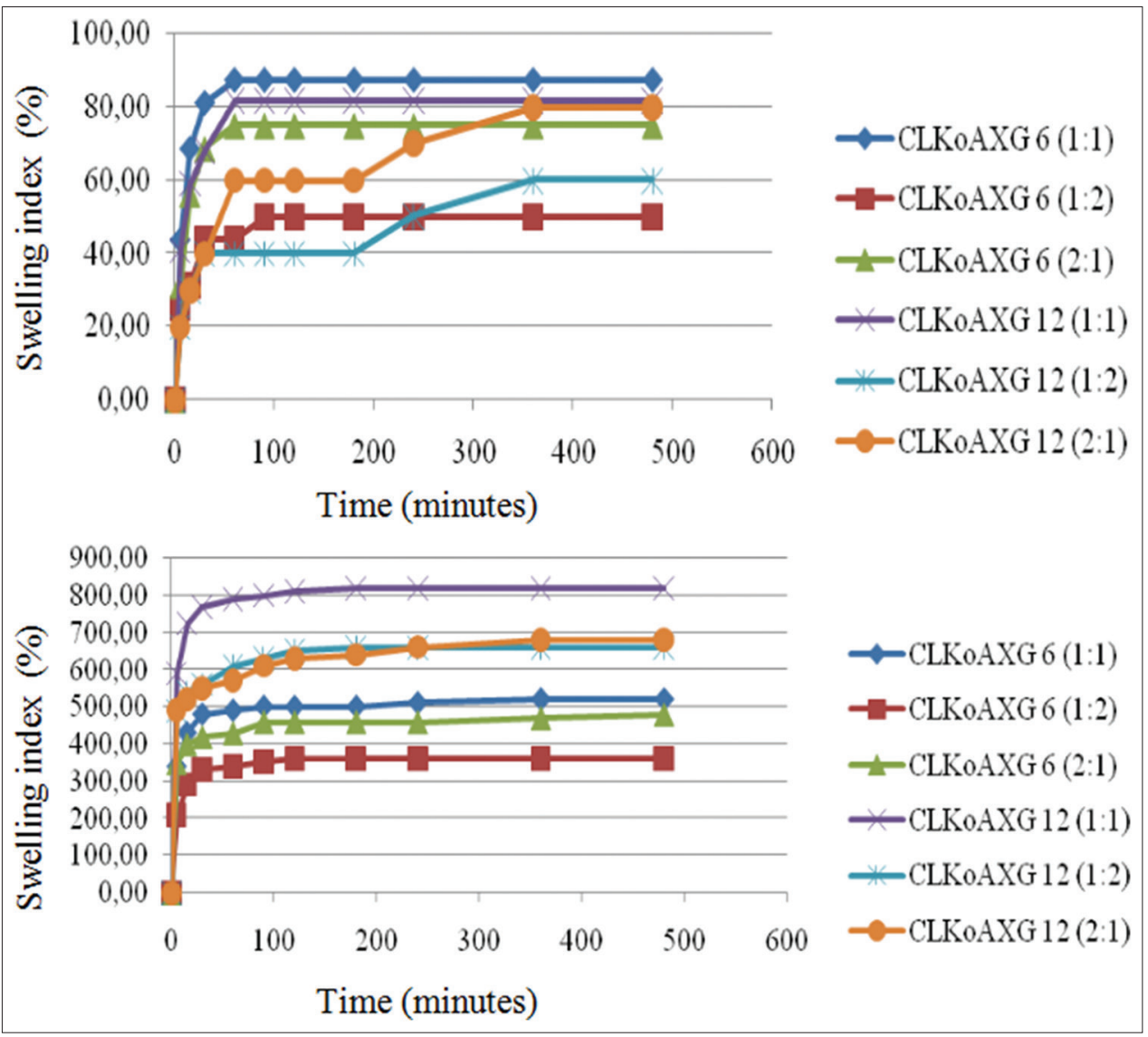

Fig. 8: Swelling index of CL Co-A-XG 
Table 6: Flowability properties of Co CLA-XG

\begin{tabular}{llll}
\hline Excipient & Flow rate $(\mathrm{g} / \mathbf{s})$ & Tapped density $(\mathrm{g} / \mathbf{m l})$ & Compressibility index $(\%)$ \\
\hline Co CLA6:XG (1:1) & 4.08 & 0.80 & 16.67 \\
Co CLA6:XG (1:2) & 4.05 & 0.82 & 18.33 \\
Co CLA6:XG (2:1) & 3.97 & 0.80 & 18.03 \\
Co CLA12:XG (1:1) & 4.17 & 0.80 & 18.03 \\
Co CLA12:XG (1:2) & 4.00 & 0.82 & 18.33 \\
Co CLA12:XG (2:1) & 4.14 & 0.82 & 18.33 \\
\hline
\end{tabular}

Table 7: Degree of substitution of CL Co-A_XG

\begin{tabular}{lll}
\hline Excipient & \% P & DS \\
\hline CLCoAXG 6 (1:1) & $1.56 \pm 0.039$ & $0.08 \pm 0.002$ \\
CLCoAXG 6 (1:2) & $1.62 \pm 0.03$ & $0.08 \pm 0.002$ \\
CLCoAXG 6 (2:1) & $1.66 \pm 0.041$ & $0.08 \pm 0.002$ \\
CLCoAXG 12 (1:1) & $2.18 \pm 0.123$ & $0.11 \pm 0.007$ \\
CLCoAXG 12 (1:2) & $2.27 \pm 0.093$ & $0.12 \pm 0.006$ \\
CLCoAXG 12 (2:1) & $2.33 \pm 0.038$ \\
\hline
\end{tabular}

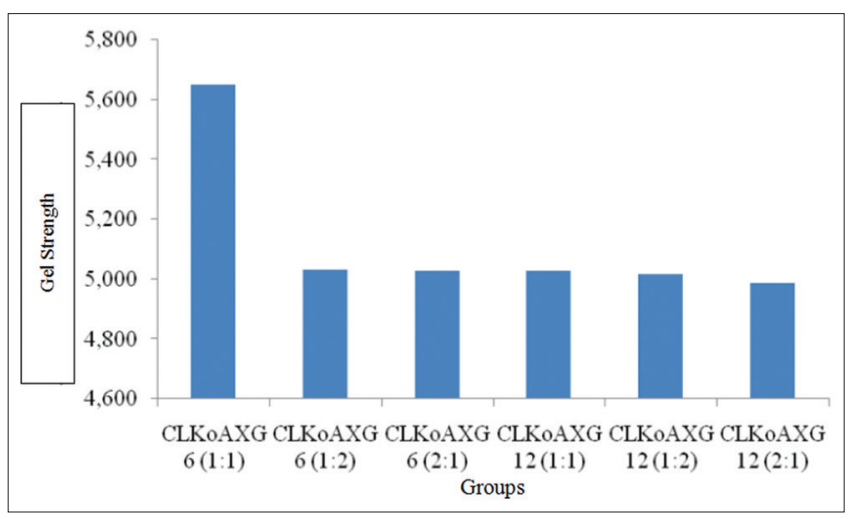

Fig. 9: Gel Strength of CL Co-A-XG

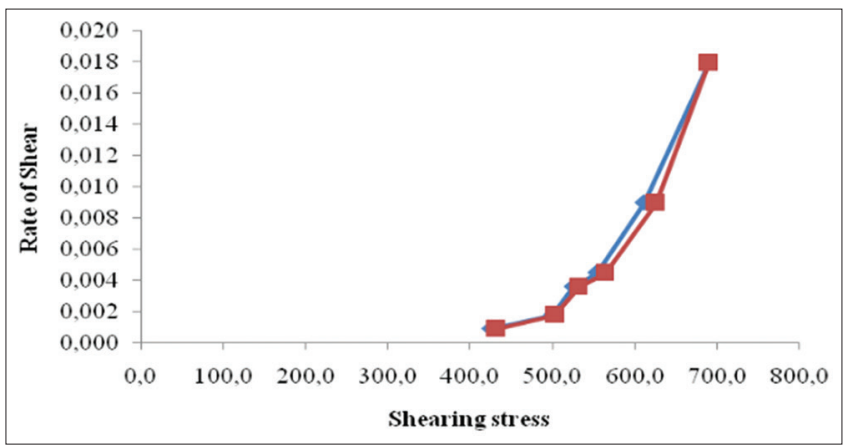

Fig. 10: Rheology of CL Co-A-XG

CL6 Co A-XG (1:1) has the highest gel strength compared with other excipients (Fig. 9)

Based on its swelling index and gel strength, CL6-Co-A-XG (1:1) is a prospective excipient for use in slow-release preparations. This is supported by the fact that a suspension of CL-Co-A-XG in distilled water exhibited pseudoplastic thixotropic flow properties; thus, CL6-Co-A-XG (1:1) is a suitable excipient for slow-release dosage forms. Rheology of CL Co A-XG is presented in Fig. 10.

\section{CONCLUSION}

Based on its swelling index and gel strength, the CL6-Co-A-XG excipient was superior to Co-CLA6-XG for use in slow-release dosage forms. Among the entire collections of crosslinked coprocessed excipients
Table 8: pH of the dispersion

\begin{tabular}{ll}
\hline Sample (1\%) & pH \\
\hline CoAXG (1:1) & $6.21 \pm 0.02$ \\
CoAXG (1:2) & $6.19 \pm 0.07$ \\
CoAXG (2:1) & $6.31 \pm 0.12$ \\
CLCoAXG 6 (1:1) & $6.39 \pm 0.05$ \\
CLCoAXG 6 (1:2) & $6.39 \pm 0.03$ \\
CLCoAXG 6 (2:1) & $6.41 \pm 0.03$ \\
CLCoAXG 12 (1:1) & $6.38 \pm 0.03$ \\
CLCoAXG 12 (1:2) & $6.43 \pm 0.03$ \\
CLCoAXG 12 (2:1) & $6.43 \pm 0.03$ \\
\hline
\end{tabular}

produced and tested, CL6 Co-A-XG (1:1) is the most suitable excipient for this purpose.

\section{ACKNOWLEDGMENTS}

All authors acknowledge the Education Ministry of Indonesia for support and PUPT Research Grants.

\section{CONFLICTS OF INTEREST}

All authors have none to declare

\section{REFERENCES}

1. Ogaji IJ, Nep EI, Audu-Peter JD. Advances in natural polymers as pharmaceutical excipients: Review article. Pharm Anal Acta 2011;3:1-16

2. Acharya K, Khatua S, Mitra P. Free radical scavenging and nos activation properties of water soluble crude polysaccharide from Pleurotus ostreatus. Asian J Pharm Clin Res 2013;6:67-70.

3. Reddy PS, Bose PSC, Sruthi V, Saritha D. Investigation of kondagogu gum to develop transdermal film of repaglinide. Asian J Pharm Clin Res 2018;11:440-5.

4. Ofori-kwakye K, Amekyeh H, El-duah M, Kipo SL. Mechanical and tablet coating properties of cashew tree (Anacardium occidentale $\mathrm{L}$ ) gum-based films. Asian J Pharm Clin Res 2012;5:62-8.

5. Carbinatto FM, de Castro AD, Cury BS, Magalhães A, Evangelista RC. Physical properties of pectin-high amylose starch mixtures cross-linked with sodium trimetaphosphate. Int J Pharm 2012;423:281-8.

6. Ferreira DD, Costa LA, Campos MI, Bispo MD, Krause LC, Macedo ML, et al. Production of xantham gum from soybean biodiesel: A preliminary study. BMC Proc 2014;8:P174

7. Disha JS, Begum MH, Shawan WM, Khatun N, Ahmed S, Islam MS, et al. Preparation and characterization of xanthan gum-based biodegradable polysaccharide hydrogels. Res J Mater Sci 2016;4:13-8.

8. Soares GA, de Castro AD, Cury BS, Evangelista RC. Blends of crosslinked high amylose starch/pectin loaded with diclofenac. Carbohydr 
Polym 2013;91:135-42.

9. Heo H, Lee YK, Chang YH. Effect of cross-linking on physicochemical and in vitro digestibility properties of potato starch. Emirates J Food Agric 2017;29:463-9.

10. Lenaerts V, Moussa I, Dumoulin Y, Mebsout F, Chouinard F, Szabo P, et al. Cross-linked high amylose starch for controlled release of drugs: Recent advances. J Control Release 1998;53:225-34.

11. Ispas-Szabo P, Ravenelle S, Hassan I, Preda M, Mateeschu MA. Structure-properties relationship in cross-linked high-amylose starch for use in controlled drug release. Carbohydr Res 2000;323:163-75.

12. Bejenariu A, Popa M, Dulong V, Picton L, Cerf DL. Trisodium trimetaphosphate crosslinked xanthan networks: Synthesis, swelling, loading, and releasing behavior. Polym Bull 2009;62:525-38.

13. Nachaegari KS, Bansal AK. Coprocessed excipients for solid dosage form. Pharm Technol 2004;14:54-8.

14. Vodackova P, Vranikova B, Svacinova P, Franc A, Elbi J, Muselik J, et al. Evaluation and comparison of three types of spray dried coprocessed excipient Avicel ${ }^{\circledR}$ for direct compression. Biomed Res Int 2018;3:1-15

15. Saha SS, Shahiwala AF. Multifunctional co-processed excipients for improved tableting performance. Expert Opin Drug Deliv 2009;6:197-208.

16. Cury BS, Klein ST, Evangelista RC. Modeling a system of phosphated cross-linked high amylosefor controlled drug release. Part 1: Synthesis and polymer characterization. React Funct Polym 2008;68:1200-6.

17. Vliegenthart JF, van de Burg YE, Bergsma J, Bleeker IP, Mijland PJ, Kamerling JP. Structural studies on methylated starch granules. Rev Starch/Starke 1988;52:40-3. 